\title{
A Pilot Study of Jugular Compression (Queckenstedt maneuver) for Cranial Movement Perception
}

Alessandra Abenavoli, PhD (Italy); Stefano Pisa, DO (Italy), BSc Ost (UK); Alberto Maggiani, DO (Italy), BSc Ost (UK), PT

From the Italian Academy of Osteopathic Medicine in Saronno, Italy.

Financial Disclosures: None reported.

Support: None reported.

Address correspondence to

Alberto Maggiani, DO, Research Department, Italian Academy of Osteopathic Medicine (AIMO), Piazza Santuario 721047 Saronno (VA), Italy Email: alberto.maggiani@aimoedu.it Submitted May 20, 2019; final revision received November 12, 2019; accepted December 16, 2019
Context: Osteopathy in the cranial field (OCF) is among the most controversial topics of osteopathic practice. The mechanism by which cranial movement (CM) occurs is poorly understood, but includes speculation that intracranial pressure can generate a movement of the cranial bones. If this model is valid, an increase in intracranial pressure produced by bilateral compression of internal jugular veins, or the Queckenstedt maneuver (Q-test), should be detectable.

Objective: To determine whether osteopaths can perceive a palpable change in $\mathrm{CM}$ when the Q-test is applied.

Methods: Blindfolded osteopaths experienced in OCF evaluated the CM of volunteers as a trained clinician applied the Q-test. The osteopaths reported any change in $\mathrm{CM}$ amplitude during 3 different 1-minute periods. The total number of variations perceived in each period (PV) by all osteopaths on all volunteers was analyzed. The Kruskal Wallis test was used to evaluate the differences between the test periods. The Mann-Whitney test was used for a pairwise comparison. Statistical significance was set at $P \leq .05$.

Results: Eight osteopaths participated in this study and evaluated the CM of 6 volunteers. A Kruskal-Wallis test of the PV between monitoring periods revealed a statistically significant difference $(P<.001)$. A Mann-Whitney Test showed there was a significant increase in PV between the compression period and the 2 other monitoring periods (base $P=.003$ and expectation $P=.009$ ).

Conclusion: Osteopaths could detect a change in the amplitude of CM after the Qtest was applied. Although this was a small-scale pilot study, our data represent a starting point for understanding whether an intracranial or an extracranial mechanism is responsible for the $\mathrm{CM}$.

J Am Osteopath Assoc. 2020;120(10):647-654. Published online September 7, 2020

doi:10.7556/jaoa.2020.119

Keywords: cranial movement, OCF, OMTh, osteopathic manipulation, Queckenstedt maneuver

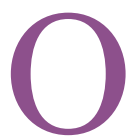
ne of the most controversial aspects of osteopathic practice is osteopathy in the cranial field (OCF). ${ }^{1,2}$ Osteopaths practicing OCF suggest that it is possible to manually perceive cranial movement (CM), or variations in the size of the cranial theca, through the movement of the cranial bones. Although not universally accepted, the evaluation and correction of $\mathrm{CM}$ dysfunctions has been reported to be effective for managing various medical conditions. ${ }^{3,4}$ 
To support the clinical applications of OCF, an understanding of the underlying mechanism responsible for the palpatory perception of $\mathrm{CM}$ is critical. The classical biomechanical model assumes a certain degree of mobility of the cranial bones at the sutural level, which persists into adulthood. ${ }^{2,5}$ Because of this mobility, osteopaths should be able to monitor changes in the volume of fluids contained in the skull, such as the cerebrospinal fluid (CSF) or cerebral blood volume (CBV). ${ }^{6}$ Externally applied forces to the skull or increases in intracranial pressure have been shown to generate a movement of the cranial bones. ${ }^{7}$ However, some osteopaths reject this biomechanical model and hypothesize that extracranial mechanisms are responsible for CM perception. ${ }^{8,9}$

Sutherland has proposed that the CM is an oscillatory movement with 6 to 15 cycles per minute, the so-called cranial rhythmic impulse (CRI). ${ }^{1,2}$ Concerning the biomechanical model, several mechanisms have been hypothesized to justify a fluctuation in the volume or pressure of CSF and blood flow that could explain the CRI. Heartbeat has been shown to move the brain and the CSF. ${ }^{10}$ Changes in arterial blood pressure - or more precisely, changes in the Traube-Hering-Mayer (THM) waves - are related to the CRI. ${ }^{11}$ THM waves, described as oscillations of blood flow velocity nonsynchronous with a ventilatory pattern, are concurrent with the perception of CM. ${ }^{11}$ Pulsatility in veins and lymphatic vessels could also contribute to CRI. ${ }^{2}$ Unbalance in the production and reabsorption of the CSF, with a subsequent variation of the CSF volume, was considered to be the process underlying the CRI for many years. ${ }^{2}$ According to the conventional theory, CSF is produced in the choroid plexus; reabsorption of CSF occurs via the arachnoid granulations into the superior sagittal sinus and through cerebral and spinal arachnoid villi, which penetrate venous sinuses and root veins. ${ }^{12-14}$ Recent research suggested that the hydrodynamics of cerebrospinal fluid were indicative of a considerable amount of CSF being absorbed by the lymphatic system, making the hypothesis of fluctuations in the volume
CFS due to the mechanism of the conventional theory less plausible. $^{15}$

The different frequencies of these processes (heartbeat, THM waves, veins, and lymphatic vessel pulsatility, unbalance in production and reabsorption of the CSF) and the difficulty of attributing the CRI to a single mechanism led to the idea that the palpation of the CM results from sutural accommodation to different pressure waves and could be the cause of poor interoperator reliability in palpation of the CM rate. ${ }^{4,16}$

If the CM biomechanical model is valid, an osteopath should perceive an increase in intracranial pressure regardless of the mechanism of CRI. The Queckenstedt maneuver (Q-test), or bilateral compression of internal jugular veins (IJV), is a technique that has been proven effective in increasing intracranial pressure. The Q-test obstructs the cephalic venous return and increases the venous blood volume in the cranial cavity, increasing consequently the intracranial pressure. ${ }^{17}$ In clinical practice, the Q-test has long been used to diagnose migraines and cranial compliance disorders such as spinal canal blockage. ${ }^{18}$

In rats, external and internal jugular veins converge to form bilateral common jugular veins (CJV). ${ }^{19}$ When changes in cerebrospinal fluid pressure induced by compressing the CJVs with the Q-test or by directly blocking the flow with bilateral clipping were compared in rats, their response to the Q-test was greater from clipping. ${ }^{20,21}$ Indeed, other collateral brain venous returns could also be obstructed during a Q-test in addition to bilateral CJV. ${ }^{20}$ Furthermore, intraventricular pressure returned to baseline when performing the Q-test for more than 30 seconds. ${ }^{20}$ This return to baseline may be indicative of the activation of compensatory mechanisms for maintaining the physiological condition of compliance of the intracranial pressure. ${ }^{15}$

The aim of this study was to determine whether osteopaths experienced in OCF could perceive a palpable change in $\mathrm{CM}$ when the Q-test was applied to volunteers. 


\section{Methods}

This study was approved by the Ethics Committee of the British College of Osteopathic Medicine in the United Kingdom (BCOM) and Accademia Italiana di Medicina Osteopatica Ethical Revision Committee.

\section{Participants}

Participants included osteopaths who had more than 2 years of clinical experience with OCF who were able to be blindfolded. Six volunteers were recruited from the student body of the Accademia Italiana di Medicina Osteopatica in Saronno, Italy. The sample size chosen was similar to that used in studies assessing interrater reliability of the CRI rhythm. ${ }^{4}$ Volunteers were included if they were 18 years of age or older, gave informed consent regarding the research objectives, and were able to remain supine without pain or discomfort for at least 30 minutes. Exclusion criteria included having bradycardia (a heart rate of less than $60 \mathrm{bpm}$ ) or any cardiovascular disease known to be affected by the Q-test on the vagus nerve.

\section{Procedures}

All volunteers were evaluated on the same morning in July 2017. CM was assessed by the osteopaths, with each volunteer in a supine position. The Q-test was applied by an experienced clinician and in the presence of an assistant. The clinician applied gentle pressure to the jugular vein for 60 seconds (Figure 1), monitored the radial pulse of the volunteers, and watched for any possible sensation of pain or discomfort. In preparation for the experiment, osteopaths underwent training to evaluate the amplitude of CM, the parameter we thought most likely to be influenced by the Q-test, and to record any perceived variation in CM.

Each osteopath was kept isolated from other participating osteopaths. Volunteers were randomized to different study rooms where each osteopath evaluated each volunteer (Figure 2). The osteopath sat at the head of the bed, which allowed proper evaluation of the volunteer's CM. Once the osteopath was correctly positioned and in contact with the volunteer head, he

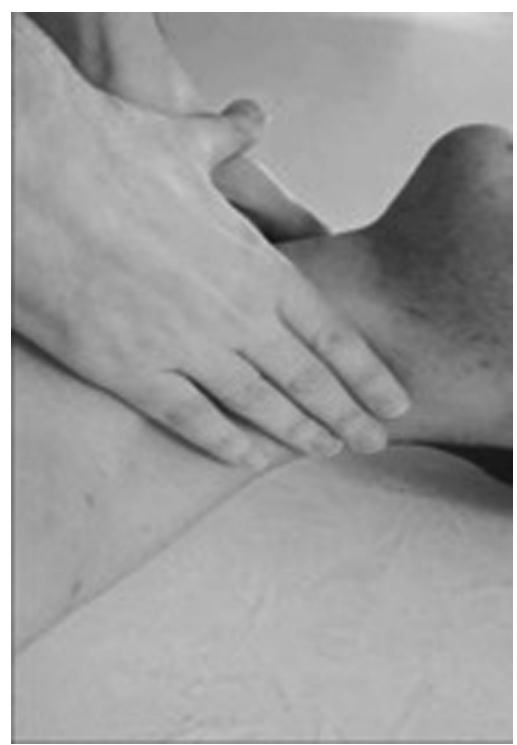

Figure 1.

Queckenstedt's maneuver.

or she was blindfolded by the assistant and was allowed a few minutes to "synchronize" with the volunteer's CM prior to evaluating the width of the CM using an arbitrary scale between 0 (no movement) and 3 (the widest movement). Each osteopath then began assessment of any change in the perception of the CM amplitude and said "increase" or "decrease" whenever they perceived a change regardless of the magnitude of the variation in $\mathrm{CM}$.

Changes in CM amplitude were recorded for 4 minutes. The time interval was chosen following a test that was performed before the trial; osteopaths reported that $\mathrm{CM}$ monitoring for more than 4 minutes caused mental fatigue, resulting in a loss of concentration that compromised their ability to monitor CM. During the first minute of CM monitoring (the "base period"), the Q-test was not applied to the volunteer. For the second minute (the "expectation period"), the Q-test was not applied, but osteopaths were informed about the "possible" application of the Q-test; this was to confound the osteopath's expectations. For the third minute (the "compression period"), the Q-test was applied by the clinician. To avoid abruptly interrupting the evaluation 


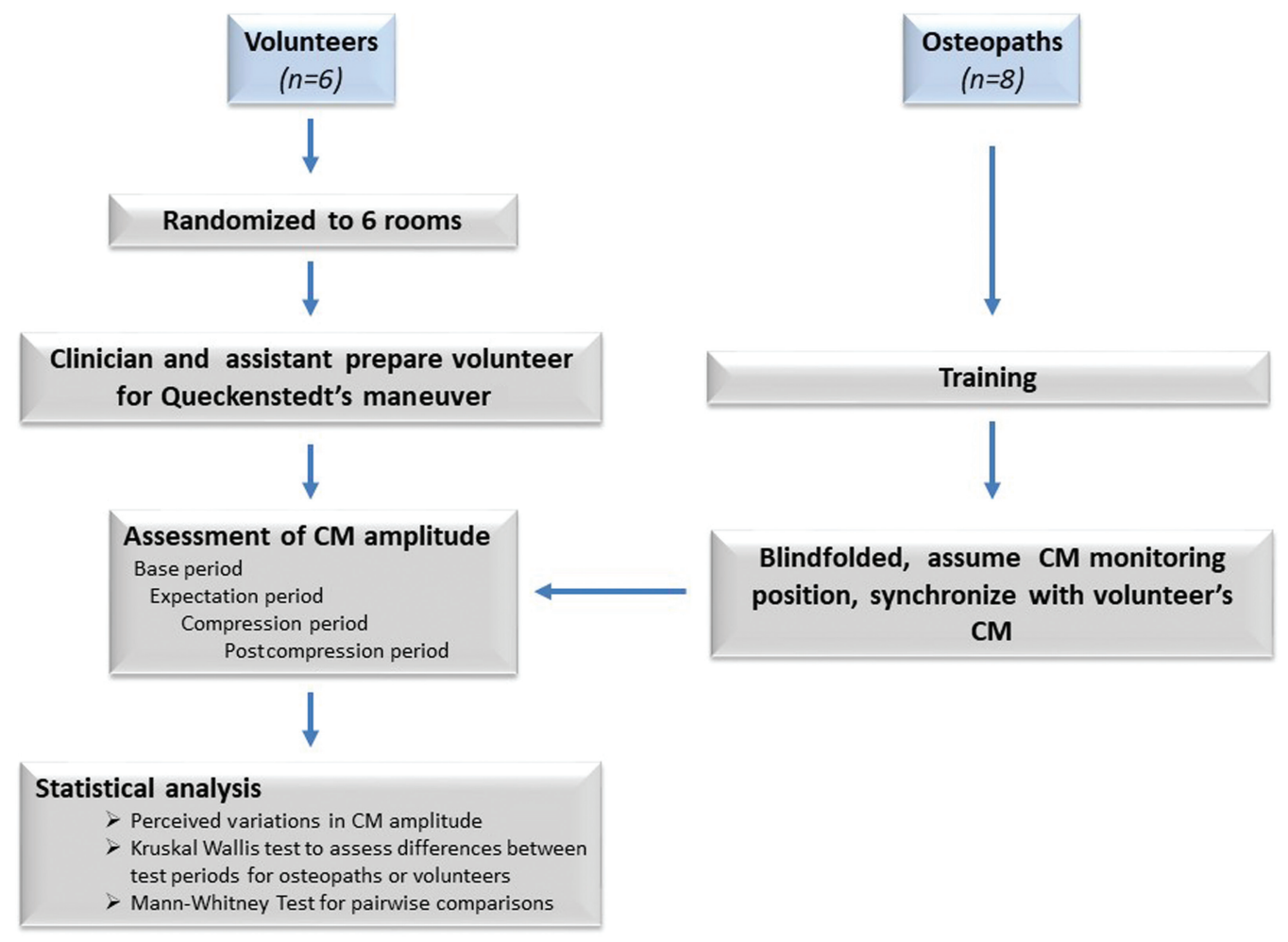

Figure 2.

Flow chart of the experimental procedure. Abbreviation: CM, cranial movement.

of CM by the osteopath, at the end of the third minute, compression was released but evaluation of CM by the osteopaths continued for a timed interval (the "postcompression period") decided by the assistant (usually 30-60 additional seconds). The changes recorded in postcompression period were not included in data analysis. Osteopaths were not informed of the use of the different monitoring periods.

To standardize the manual pressure for the Q-test, a weighting scales was positioned under the head of the volunteers and set to a maximum value a pressure of 300 grams, which in our view is sufficient to collapse the jugular veins.

\section{Statistical analysis}

The number of perceived variations (PV) in the amplitude of CM in the base, expectation, and compression periods were the primary outcome measures analyzed.
For each monitoring period, PV was calculated by summing the variation perceived by the osteopaths for all the 6 volunteers, which resulted in 48 independent measurements of PV for the test period. The Kruskal Wallis test was used to assess differences between test periods, osteopaths, or volunteers. The Mann-Whitney test was used for a pairwise comparison. Statistical significance was set at $P \leq .05$.

\section{Results}

Demographic data for the osteopaths and volunteers who took part in this study are given in Table 1. Eight osteopaths and 6 volunteers participated in this study; no participant satisfying the eligibility criteria was excluded. Figure 3 shows a typical recording for a single volunteer: each horizontal line represents the evaluation of a $\mathrm{CM}$ by a single osteopath. Each 
Table 1.

Epidemiological Data for Osteopaths and Volunteers ${ }^{a}$

\begin{tabular}{lll} 
Characteristics & $\begin{array}{l}\text { Osteopaths } \\
(\mathbf{n = 8})\end{array}$ & $\begin{array}{l}\text { Volunteers } \\
(\mathbf{n}=6)\end{array}$ \\
\hline Age & $31.13(6.49)$ & $21.17(4.31)$ \\
\hline Sex (F/M) & $3 / 5$ & $2 / 4$ \\
\hline BMI & $22.63(1.41)$ & $22.83(2.71)$ \\
\hline Experience (y) & $4.25(2.54)$ & $\ldots$ \\
\hline Health status & No known & No known \\
& pathologies & pathologies \\
\hline Medication & None & None \\
\hline Smokers & 3 & 2 \\
\hline
\end{tabular}

a Values are mean (SD).

deviation from baseline represents a change in perception of CM amplitude by the osteopath. A downward deviation represents a decreased perception of CM amplitude, and an upward deviation represents an increase in perception of $\mathrm{CM}$ amplitude. In the example given (Figure 3), no CM amplitude variations were detected in the base period. During the expectation period, osteopaths \#3 and \#7 detected a decrease in CM amplitude. In the compression period, 3 osteopaths (\#3, \#4, and \#5) perceived an increase followed by a decrease in CM amplitude, 2 osteopaths (\#2 and \#7) perceived an increase in $\mathrm{CM}$ amplitude that persisted for the entire period, 2 osteopaths (\#1 and \#8) perceived a decrease followed by an increase in CM amplitude. Finally, osteopath \#6 did not perceive any variation of CM amplitude in any period. For this volunteer, the PV (Figure 3) was 0 for the base period, 2 for the expectation period, and 12 for the compression period.

The PV variable represents the contribution of all osteopaths summed for all of the volunteers (Table 2 and Figure 4). There were $2 \mathrm{PVs}$ in the base period (2 osteopaths reassessed the initial evaluation, but this was considered as a variation in the perceived CM amplitude). During the expectation and the compression period, 34 and 60 PVs were recorded, respectively

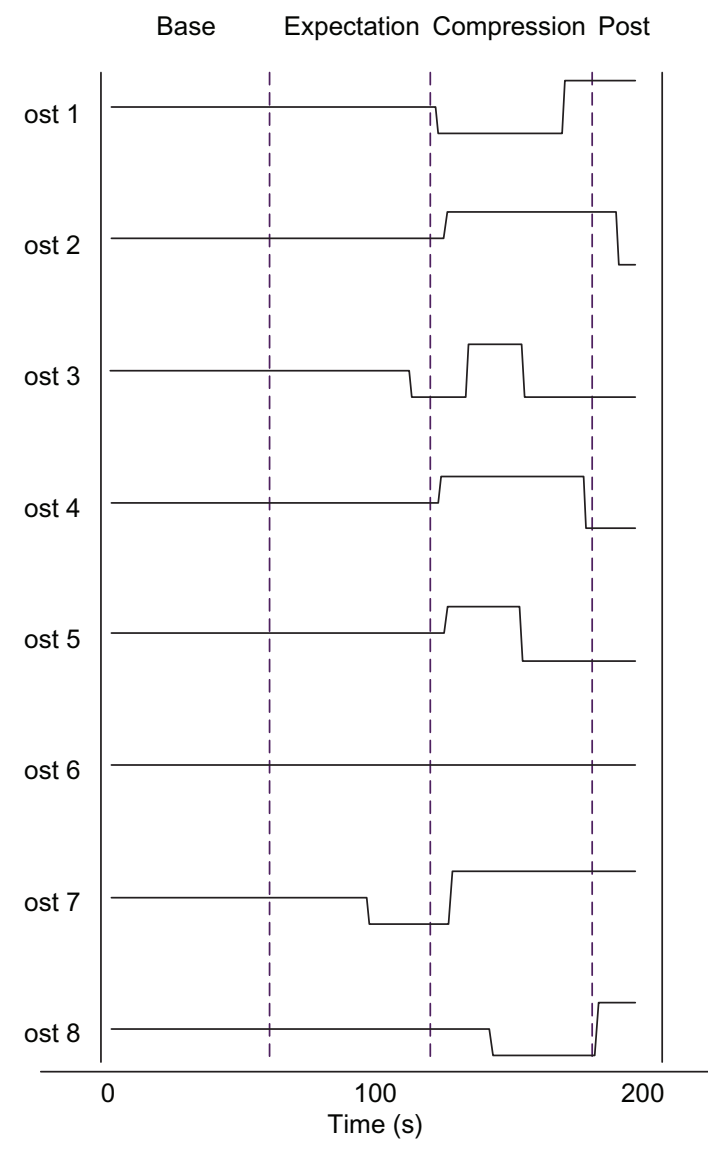

Figure 3.

Typical experimental recording for a volunteer. Each line is the evaluation of an individual osteopath (ost 1 to ost 8) for a single volunteer. Each deflection from this line represents an increase in amplitude (upward deflections) or a decrease in amplitude (downward deflections).

(Table 2 and Figure 4). Using the Kruskal-Wallis test, the variation in PV between the 3 test periods showed significant differences $(\mathrm{H}=64.337, \mathrm{df}=2 ; P<.001)$. A Mann-Whitney Test analysis showed a significant increase in PV between the compression period and the base and expectation periods $(P=.003$ and $P=.009$, respectively) and a significant difference between the base period and the expectation period $(P=.003)$. There was no statistical significance for PV between different osteopaths (Kruskal-Wallis test; $\mathrm{H}=7.272$; $\mathrm{df}=7 ; P=.401)$ or between different volunteers evaluated (Kruskal-Wallis test; $\mathrm{H}=2.951 ; \mathrm{df}=5 ; P=.791$ ). 


\section{Discussion}

We used the Q-test to monitor variations in the perception of cranial movement by osteopaths. We found a significant effect of the compression period in comparison to the expectation period and the base period $(P=.003$ and $P=.0009)$. This finding indicates that the osteopaths perceived a variation in the cranial movement during the jugular compression maneuver and distinguished it from the expectation effect. This finding suggests that the mechanism responsible for the palpatory sensation could be an intracranial, rather than an extracranial, phenomenon. Although the Q-test was shown to be effective in increasing intracranial pressure, anastomotic variants between the external jugular and internal jugular can be found; the Q-test could generate an extracranial effect if applied downstream of an anastomosis inducing a possible epicranial

Table 2.

Perceived Variations (PV) in the Cranial Movement (CM) Amplitude Analysed During the 3 Test Periods

Period

(A)

\begin{tabular}{lcccc} 
(A) & Total & Median & Q1 & Q3 \\
\hline Base & 2 & 0 & 0 & 0 \\
\hline Expectation & 34 & 1 & 0 & 1 \\
\hline Compression & 60 & 1 & 1 & 1 \\
\hline
\end{tabular}

\section{(B)}

Kruskal Wallis ${ }^{\mathrm{b}}$

\begin{tabular}{ll}
\hline Period & $P<.001$ \\
\hline Osteopaths & $P=.401$ \\
\hline Volunteers & $P=.791$ \\
\hline
\end{tabular}

(C)

\begin{tabular}{lc} 
(C) & Mann-Whitney Test $^{\mathrm{c}}$ \\
\hline Base vs expectation & $P=.003$ \\
\hline $\begin{array}{l}\text { Expectation vs } \\
\text { compression }\end{array}$ & $P=.003$ \\
\hline Base vs compression & $P=.009$
\end{tabular}

a PV in the CM amplitude analysed over the 3 test periods. Median, first and third quartile (Q1, Q3) are shown.

b Kruskall Wallis differences between test periods, osteopaths, or volunteers.

c Mann-Whitney test for pairwise comparison.

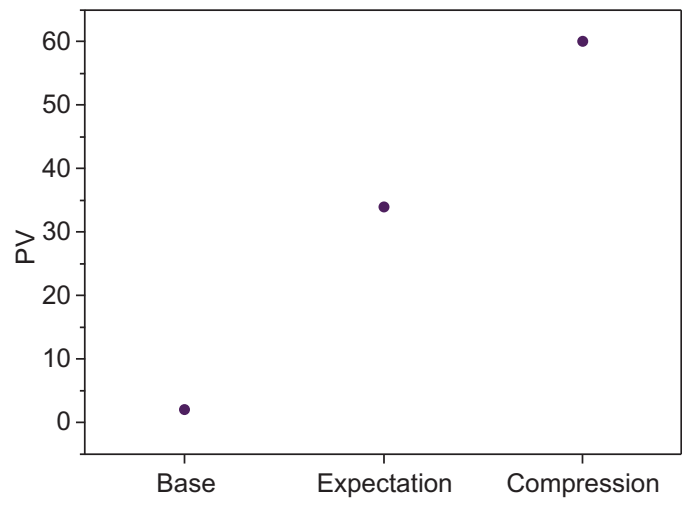

Figure 4.

Total number of perceived variations (PV) in the cranial movement amplitude over the 3 test periods.

tactile stimulus. ${ }^{22}$ A critical variable in our study was represented by the compression of the jugular itself. Although the Q-test was performed by experienced clinicians and the amount and placement of the pressure was coordinated, there is a possibility that different clinicians were not always equally effective in reducing cranial drainage. Future studies should include a measure of the effectiveness of jugular compression, possibly using imaging techniques.

The limited duration of the 3 evaluation periods could be seen as a limitation; however, it proved difficult and inappropriate to increase the evaluation time of each period because of a resulting decrease in the concentration of the osteopaths. Moreover, maintaining the Q-test for more than 30 seconds does not improve perception of an increase in intracranial pressure. ${ }^{20}$ Therefore, the choice of 3 periods, each lasting 1 minute, seemed most appropriate for this study.

In designing this experiment, we preferred not to assume a direction (increase or decrease) for a variation in $\mathrm{CM}$ amplitude because it is not possible to predict how an increase in intracranial pressure could modify the hypothesized mechanisms responsible for the CRI. Some osteopaths perceived an increase in amplitude, while others perceived a decrease; therefore, the significant variable was the perception of a change in $\mathrm{CM}$ rather than an increase or decrease in CM amplitude. 
The perception of a change indicates a disturbance of the system being evaluated. Moreover, the literature ${ }^{23}$ suggests that assessing movement is more reliable than assessing the direction of movement.

Our data also found a significant difference when comparing the expectation with the base periods. This observation confirms the effectiveness of blindfolding the osteopaths with respect to compression: 7 out of 8 osteopaths perceived changes in the amplitude during the second phase of the experiment. Also, the observation could show that the osteopaths' expectations of having to perceive something generated false positives. Kasparian et $\mathrm{al}^{23}$ found similar results using a mechanical model.

In our study, osteopaths were asked to detect possible variations in the width of the CM only from the expectations phase. In the base period, osteopaths were asked to evaluate only the amplitude but they were not expecting any changes. For this reason, the base period does not constitute an appropriate control for the expectation period in term of fluctuations detected.

There are no data in the literature as to the reliability of using amplitude to evaluate CM; this is because the rhythm parameter seems to be more objectively measurable. Reliability in the evaluation of the rhythm of the $\mathrm{CM}$ is poor. ${ }^{4}$ In a clinical setting, the assessment of the extent of the CM and its possible variation following treatment is more widespread. For educational purposes, a numerical evaluation of the CM is sometimes used, considering 0 as the absence of movement and 3 as the maximum perceptible movement. This kind of evaluation is highly subjective, and in our study, the reliability of the initial evaluation of the amplitude was found to be "slight agreement" on the Landis and Koch scale $(\mathrm{k}=0.0549){ }^{24}$

\section{Conclusions}

This study suggests that changes in the cranial movement fluctuations can be perceived by an osteopath when the jugular compression Q-test is applied. Moreover, our results suggest that perception of an inducted intracranial phenomenon was reproducible between osteopaths. Although this was a small-scale pilot study, our data represent a starting point for understanding if an intracranial or an extracranial mechanism is responsible for the $\mathrm{CM}$.

\section{Author Contributions}

All authors provided substantial contributions to conception and design, acquisition of data, or analysis and interpretation of data; all authors drafted the article or revised it critically for important intellectual content; all authors gave final approval of the version of the article to be published; and all authors agree to be accountable for all aspects of the work in ensuring that questions related to the accuracy or integrity of any part of the work are appropriately investigated and resolved.

\section{References}

1. Sutherland WG, Gregory MK. The Cranial Bowl: A Treatise Relating to Cranial Articular Mobility, Cranial Articular Lesions and Cranial Technic. Free Press Company; 1939.

2. Ferguson A. A review of the physiology of cranial osteopathy J Osteopath Med. 2003;6(2):74-84.

3. Jäkel A, Von Hauenschild P. Therapeutic effects of cranial osteopathic manipulative medicine: a systematic review. J Am Osteopath Assoc. 2011;111(12):685.

4. Guillaud A, Darbois N, Monvoisin R, Pinsault N. Reliability of diagnosis and clinical efficacy of cranial osteopathy: a systematic review. PloS One. 2016;11(12):e0167823. doi:10.1371/journal.pone. 0167823

5. Cook A. The SBS revisited-the mechanics of cranial motion. J Bodyw Mov Ther. 2005;9(3):177-178.

6. Moskalenko YU, Weinstein G, Masalov I, Ryabchikova VS, Panov A Andreeva Y. Multifrequency REG: fundamental background, informational meaning and ways of data analysis and automation. Am J Biomedic Eng. 2012;24(2):163-174. doi:10.5923/j.ajbe.20120204.03

7. Seimetz CN, Kemper AR, Duma SM. An investigation of cranial motion through a review of biomechanically based skull deformation literature. Int J Osteopath Med. 2012;15(4):152-165

8. McGrath MC. Viewpoint. J Osteopath Med. 2003;6(2):84-86.

9. Gabutti M, Draper-Rodi J. Osteopathic decapitation: Why do we consider the head differently from the rest of the body? New perspectives for an evidence-informed osteopathic approach to the head. Int J Osteopath Med. 2014;17(4):256-262.

10. Greitz D, Wirestam R, Franck A, Nordell B, Thomsen C, Ståhlberg F. Pulsatile brain movement and associated hydrodynamics studied by magnetic resonance phase imaging. Neuroradiol. 1992;34(5):370-380.

11. Nelson KE, Sergueef N, Lipinski CM, Chapman AR, Glonek T. Cranial rhythmic impulse related to the Traube-Hering-Mayer oscillation: comparing laser-Doppler flowmetry and palpation. J Am Osteopath Assoc. 2001;101(3):163-173.

12. Cutler RWP, Page L, Galicich J, Watters GV. Formation and absorption of cerebrospinal fluid in man. Brain. 1968;91(4):707-720.

13. Bardan G, Plouraboué F, Zagzoule M, Baledent O. Simple patient-based transmantle pressure and shear estimate from cine 
phase-contrast MRI in cerebral aqueduct. IEEE Transactions Biomedic Eng. 2012;59(10):2874-2883.

14. Spector R, Snodgrass SR, Johanson CE. A balanced view of the cerebrospinal fluid composition and functions: focus on adult humans. Exp Neurol. 2015;273:57-68. doi:10.1016/j.expneurol.2015.07.027

15. Miyajima, M, Arai $\mathrm{H}$. Evaluation of the production and absorption of cerebrospinal fluid. Neurologia Medico-Chirurgica. 2015;55 (8):647-656. doi:10.2176/nmc.ra.2015-0003

16. Chaitow L. Viewpoint. J Osteopath Med. 2003;6(2):86-88

17. Chou $\mathrm{CH}$, Chao AC, Lu SR, Hu HH, Wang SJ. Cephalic venous congestion aggravates only migraine-type headaches. Cephalalgia. 2004;24(11):973-979.

18. Chou CH, Fuh JL, Wang SJ, Hu HH, Wu JC, Cheng YT. Queckenstedt's test headache response is associated with increased jugular venous flow volume during migraine attack. Ultrasound Med Biol. 2011;37(1):23-28.
19. Szabó K. The cranial venous system in the rat: anatomical pattern and ontogenetic development. Anat Embryol, 1990;182(3):225-234.

20. Pearce JMS. Queckenstedt's manoeuvre. J Neurol Neurosurg Psychiatry. 2006;77(6):728-728.

21. Chou CH, Doong ML, Fuh JL, Wu JC, Wang SJ. Queckenstedt's test affects more than jugular venous congestion in rat. PloS One. 2013;8 (3):e59409

22. Mortazavi MM, Tubbs RS, Riech S, et al. Anatomy and pathology of the cranial emissary veins: a review with surgical implications. Neurosurgery. 2011;70(5):1312-1319.

23. Kasparian H, Signoret G, Kasparian J. Quantification of motion palpation. J Am Osteopath Assoc. 2015;115(10):604.

24. Landis JR, Koch GG. The measurement of observer agreement for categorical data. Biometrics. 1977;33(1):159-174.

() 2020 American Osteopathic Association 